\title{
Los derechos humanos como desafío a la política
}

Los derechos humanos son derechos y deberes fundamentales que expresan la posición de principio de los hombres en la sociedad, y su relación con el Estado en que viven.

Los derechos humanos, en general, están determinados bajo los nombres de derechos cívicos, derechos fundamentales, las constituciones de los estados. Además, han sido fijados en diferentes convenciones de la ONU.

Como todas las formas de derecho, los derechos humanos también tienen sus raíces, en último término, en las condiciones de existencia económica de la sociedad y expresan los intereses fundamentales de grupos sociales.

Los derechos humanos, por consiguiente, no son derechos de carácter eterno, innatos al hombre como tal, ni son anteriores al Estado ni se hayan sobre éste. Son más bien producto del desarrollo histórico del Estado y del derecho, corresponden a las condiciones de la sociedad, cambian con ella y la sociedad misma los cambia. El contenido concreto de los derechos humanos, es siempre resultado y expresión de la lucha de los grupos sociales y de la correlación de fuerzas.

Los derechos humanos se basan, por consiguiente, en intereses: Sin embargo solo los intereses no justifican un derecho; es preciso que haya un derecho subjetivo correspondiente. Para justificar un derecho, se necesita a alguien a quien se le pueda obligar a cumplir tal derecho. Los derechos humanos tienen pues como correlativo; los deberes. Por eso es necesario que existan deberes humanos correspondientes.

Los derechos humanos no son ningún regalo que los hombres se ofrecen unilateralmente o en forma de reciprocidad: son un reconocimiento que los hombres se deben los unos a los otros. 
Los derechos humanos no se permite que sean realizados por reclamaciones morales, sino que su realización solamente es posible en el contexto de un derecho democráticamente autorizado y de una seguridad jurídica garantizada por un poder legítimo.

Los derechos humanos precisan de su formulación y reconocimiento como tales derechos, de tal modo que una política referente a los derechos humanos sería tanto necesaria como incompleta, mientras en esta Tierra todos los hombres no tengan, por lo menos, derechos cívicos democráticos y sociales. Sin la existencia de los últimos los derechos del hombre serán extremadamente frágiles a la vez que una expresión de deficiencia.

Ocuparse de los derechos humanos, significa a la vez tener en cuenta la existencia de derechos cívicos.

Los derechos humanos tienen menos peso que los derechos cívicos porque no son reclamables. Son reclamables los derechos sólo a nivel estatal nacional.

Los estados nacionales son los únicos actores internacionales capaces de actuar: ellos dictan el derecho, cuidan de los derechos y pueden ceder los derechos. (por ej. La comunidad europea, la Corte de Justicia Internacional).

La forma política del Estado Nacional en la historia hasta ahora es la única forma conocida en que pueden ser realizadas las libertades liberales, el derecho legitimado democráticamente, así como la seguridad social. De ahí resulta la necesidad al considerar los derechos humanos pensar de nuevo acerca del Estado Nacional, en el contexto de la globalización. Por ejemplo, la libertad política no es un derecho humano, no hay un derecho a quedarse en un país para siempre fuera de su nacimiento. Además no es posible la participación en elecciones en otro país, ya que es un derecho reservado a los ciudadanos.

Las posiciones contrapuestas entre los globalistas que exigen un estado mundial homogéneo, y los comunitaristas que rechazan todo estado mundial, habría que ser superado por una posición intermedia (hay algunas ideas primeras en Canadá, Israel y los EE UU).

La democracia política y la concepción moderno de los derechos cívicos y humanos, individuales y sociales, universales e intransferibles, se formaron juntos en un proceso histórico de desarrollo que comenzara en la antigüedad.

Ideas acerca de la dignidad de la persona humana y de un orden político (vale decir, de podeì) justo, ligado a valores morales, estuvieron difundidos y los hay en todas las civilizaciones.

Más, su despliegue a derechos y normas firmemente definidas y formuladas, es solamente el resultado de aquel proceso histórico que condujo a la sociedad civil. 
Los derechos humanos cívicos fueron los derechos de una sociedad cuyo postulado de libertad e igualdad coincidió con la inviolabilidad de la propiedad privada de los medios de producción.

La revolución de las fuerzas productivas en el contexto de la industrialización creó por primera vez en la historia humana, la posibilidad material para un desarrollo libre de cada uno, condición sine qua non para el desarrollo libre de todos los hombres.

Sin embargo la posibilidad material ni llevó ni lleva necesariamente a la posibilidad real. Para la realización de potencias económicas, es precisa la actuación política.

La política como fenómeno social está ligada a la lucha por alcanzar los intereses de grupos sociales, dentro del estado y, con su apoyo, frente a la sociedad y otros estados.

El sistema de relaciones políticas forma la base para el desarrollo de la política práctica de grupos de interés. Es decir que la lucha política consciente y organizada tiene como objetivo el logro y aseguramiento de los intereses respectivos.

Como los intereses fundamentales de grupos sociales pueden ser conquistados solamente con el apoyo de la política, esta penetra todas las esferas de la vida social. La política es una instancia en la cual debe haber gran responsabilidad dentro de una situación político-mundial conflictiva.

Las distintas posturas respecto a derechos humanos tienen su base entre fuerzas políticamente distintas, como también entre representantes de distintas culturas. Los siguientes puntos es posible que puedan tornarse en tópicos de discurso:

La relación entre los principios de los derechos humanos universales y los valores de culturas particulares.

La contradicción entre el principio de la no intervención en los asuntos internos de otros países y el principio de la condena de la violación de los derechos humanos.

El método de lucha en contra de las violaciones de derechos humanos: transformación o cambio por la vía de la cooperación o por medio del boicot.

Digamos que en la práctica mundial, la elección de los métodos de la condena de la violación de los derechos humanos, está influenciada por motivaciones económicas y/o políticas de Estado, así como por sus intereses. Esto lo observamos sobre todo, respecto al llamado primer mundo, en que hay estados que definen la elección de los métodos. (véase la conducta nula que se tiene, ya sea en el caso de China o Cuba.) 
El debate de los derechos humanos pertenece a un discurso jurídico intercultural.

Mientras el discurso de los derechos humanos se lleva en primer lugar por europeos y norteamericanos, las otras naciones pueden tener una nueva forma de imperialismo: el de la cultura jurídica.

La posición especial del discurso europeo-norteamericano acerca de los derechos humanos tiene que ponerse en relación con el discurso intercultural, desde tres ámbitos concretos: en la historia, en la teoría y en la praxis jurídica.

\section{Historia Jurídica}

Por un lado se encuentra casi todos los ordenamientos jurídicos, formas de derechos humanos; y por otro, en la cultura europea, que desarrolló temprano un concepto universal de hombre (los griegos: capacidad racional/aptitud de razón; antiguos israelitas: igual imagen de dios). Existió por siglos una desigualdad jurídica (esclavitud, guerras religiosas).

\section{Teoría Jurídica.}

Una argumentación extratemporal, es decir, la que no se remite a una justificación de una situación concreta y a una cultura jurídica particular, puede desarrollar ideas que estén abiertas para las diferencias culturales.

\section{Praxis Jurídica}

Hay principios universales que deben ser realizados cautelosamente. Es observable que culturas diferentes reciben un derecho que debe ser objeto de un proceso de aprendizaje, incluso las culturas europeo-norteamericanas. En este proceso de aprendizaje, debe ser permitido enseñar la idea de los derechos humanos universales junto con la propia cultura jurídica, pues una comunidad jurídica internacional no disuelve los estados particulares o singulares. La universalidad de principios generales no significa ninguna uniformidad: el derecho a la particularidad sigue existiendo.

Los derechos humanos son una instancia, una institución jurídica independiente de las culturas. Sin embargo, se encuentran dentro de ellos derechos dependientes de la cultura. Más la dependencia cultural no consiste en la fundamentación de la legitimación de los derechos humanos, sino en la configuración específica de ellos.

Consideramos que la idea fundamental de los derechos humanos se puede imponer a otras culturas, pero no más que esta idea fundamental: la inviolabilidad de cada persona, recíprocamente reconocidas. 
Para la configuración de los derechos humanos queda un alto grado de libertad. Por eso es que los derechos humanos contienen la fuerza de una visión futurista.

La globalización está conduciendo a una humanidad que, tanto dentro del Estado como interestatalmente, reconoce las mismas condiciones jurídicas elementales y que, por otro lado, no se deja dominar por una sola cultura.

Los derechos humanos permiten a la humanidad una identidad dentro de la diversidad.

La configuración y garantía de los derechos humanos son un asunto interno de cada Estado. Sólo cuando una violación masiva y sistemática de los derechos humanos en un Estado lleva a la amenaza de la paz, de la seguridad interna, es posible que sean aplicadas medidas internacionales.

De ahí resulta la responsabilidad muy alta para las fuerzas políticas actuantes en el ámbito nacional. La configuración y protección de derechos humanos, es un problema atinente a las relaciones políticas de poder así como de las condiciones materiales en un país determinado.

Más ha de considerarse que, a nivel nacional, esencialmente no se trata de la libertad del Estado y la sociedad, sino de libertad en la sociedad y el estado, de incluir multifaceticamente el desarrollo de la sociedad y del Estado, de la cooperación amplia de cada uno en el desarrollo de la sociedad como tal, y así, también de su personalidad.

En la República Federal de Alemania actualmente se encuentra en el orden del día la salvación del Estado Nacional. Esto no es un problema de derecho constitucional, sino uno de la política del poder. Si la política y la sociedad se ponen de acuerdo con el desempleo masivo, el Estado social será sobrecargado. Como algo necesario se muestra el reconocimiento de derechos humanos que no se encuentran en la constitución:

- El derecho al trabajo y el derecho del obrero de obtener propiedad en el producto de su obra.

También el cuidado de los derechos humanos elementales, hay que realizarlo en la República Federal Alemana, como lo demuestra el segundo informe acerca de derechos fundamentales de 1998.

En este informe, se señalan actuaciones en contra de la ley fundamental por parte del gobierno, de autoridades y de la justicia, hasta la Corte Constitucional Federal. Entre otros señalamientos está la violación de la prohibición de la retroactividad por infracciones que fueron legales en un país determinado; un principio que tiene vigencia limitada para actuaciones realizadas en la República Federal Alemana. 
La comisión de la ONU para la abolición de la discriminación racial (CERD) también formuló varias exigencias respecto a la ley para extranjeros y su aplicación en la aclaración de delitos racistas cometidos especialmente por la policía, y a la praxis del asilo. En el año de 1999 Alemania tendrá que presentar un "Informe intermedio".

$\square$ En la lucha contra el racismo, en Alemania habría necesidad de:

$\square$ Reducir las bases institucionales en que se fundamenta el racismo

口 Abolir la ley de extranjería

$\square$ El derecho de asilo

$\square$ Participación política de los extranjeros

口 Reforma del derecho de nacionalidad

$\square$ Aceptación de una ley anti-discriminatoria

$\square$ Penalizar delitos por motivos racistas

口 Prohibir la propaganda y organización racista

Ha de apoyarse por ejemplo el acuerdo modelo de empresa del consorcio Thyssen Acero Duisburg que contiene:

a) Promoción de la competencia lingüística

b) Promoción de la competencia de actuar en grupos de trabajo (teams) multiculturales y entrenamiento multicultural para fuerzas dirigentes, miembros de la empresa y aprendices.

c) Discusión de asuntos específicos

d) Información para fuerzas dirigentes alemanas acerca de la política frente a extranjeros.

e) Ayuda a familiares

La reducción de una estrategia de derechos humanos simplemente a medidas económicas y diplomáticas, es miope. Para el logro, en escala mundial de los derechos humanos, es necesaria también la movilización de la fuerza de las ideas. Ciertamente se ha de combatir la pobreza, pero es necesaria también la fe en los ideales y el coraje de intervenir cuando cualquier ser humano sea oprimido, torturado, asesinado.

En ese ámbito se encuentra también una tarea para la pedagogía y, con ésta, una correspondiente política de enseñanza. Para garantizar los derechos humanos en forma duradera se precisa de una enseñanza de los mismos. Por esta razón la ONU declaró el período de 1995 a 2004 como la década de la enseñanza de los derechos humanos. Esta enseñanza se debería incluir al sistema formal educacional. 
La ONU recomendó que las prioridades de la enseñanza de los derechos humanos sean determinadas por cada Estado, según sus condiciones concretas.

En ese contexto hay también una discusión en torno al objetivo educacional de la ciudadanía global. Este concepto es distinto al concepto jurídico, se refiere a la disposición de aprender acerca del mundo, de participar en él, de ser responsable por él y de luchar por él.

En el fondo se trata de ulterior desarrollo de la pedagogía del ambiente y de la paz, así como de la enseñanza intercultural. Como objetivos de la enseñanza se ven, entre otros, los siguientes:

a) La insensibilidad a la injusticia social

b) La capacidad de tener pensamiento crítico

c) La capacidad de solucionar conflictos pacíficamente

d) El desarrollo de la comprensión, apertura ante el mundo y capacidad de cooperación.

El desarrollo de una actividad tanto global y tanto racista como internacionalista, a la vez es posible solo bajo la condición de una sana autoestima colectiva $y$ de una identidad propia debidamente asegurada.

El objetivo global de la ciudadanía global debería penetrar todas las ramas escolares, teniendo en cuenta que no son tantos los objetivos de enseñanza cognitivos, sino los que son decisivos.

Investigadores de asuntos juveniles comprobaron que en sociedades industriales por un lado hay una fuerte decepción respecto a la política y a los partidos. En cambio, por otro lado, las organizaciones orientadas a los derechos humanos gozan de una alta confianza entre los jóvenes. Una alta orientación egoísta corre pareja con una elevada disposición al compromiso social. La condición previa para eso sería que este compromiso produzca placer y lleve algo para el joven.

Una educación en derechos humanos puede partir de esta relación entre orientación egoísta y social, educación que puede contener conocimientos de orientación y perspectivas de corto plazo para los "niños de la libertad" para no dejarlos bajo las condiciones de la individualización y globalización sin sostén y sin esperanza. Los derechos humanos y la idea de ellos, pueden ser una especie de brújula en el remolino de la modernidad; son quizás, la última "gran narración" de la libertad que puede sobrevivir con al fuerza del desarrollo cultural y político sostenible.

La educación de derechos humanos tendría que tomar en cuenta los siguientes aspectos: 
a) Desarrollo de una conciencia de libertad y disposición, de lucha por la defensa de los derechos humanos.

b) Desarrollo de la conciencia de que la protección de los derechos humanos es algo acabado (enseñar el desarrollo histórico partiendo de los derechos humanos clásicos sobre los derechos a prestaciones sociales hasta los derechos culturales.)

c) Hacer visible la lucha por la validez universal de los derechos humanos.

d) Desarrollo de la conciencia de responsabilidad (derechos y deberes humanos)

e) Enseñanza de la tolerancia.

A la vez, no se debe exagerar una enseñanza de los derechos humanos. Si bien es cierto que esta enseñanza es irrenunciable, se debe reconocer que no tiene una influencia exclusiva para el desarrollo de la conciencia acerca de los derechos humanos.

Además se observa que pedagogos de los derechos humanos se ven confrontados con un problema fundamental, a saber, el desempleo masivo.

La relación entre derechos de libertad y el derecho al trabajo $\longrightarrow$ cómo dijeran los revolucionarios de 1848: el derecho al sostenimiento a la existencia propia. - tiene que ser un tema fundamental de la discusión antes de que haya observancia de los derechos humanos en forma adecuada. "Los niños de la libertad (en las naciones industriales) se tornan siempre más en niños de miedo. Este miedo devora la disposición fundamental de actuar responsable y tolerantemente, y aumenta aquellas fuerzas dirigidas a la estrategia y de la auto afirmación de un egoísmo desesperado".

El debate acerca de los derechos humanos lleva en sí un elemento visionario, que puede contribuir tanto a la orientación como al encuentro de identidad de los seres humanos. La idea de los derechos humanos ha movilizado por siglos a los hombres; y sigue haciendo hasta hoy, frente a las numerosas ideas que aún quedan incumplidas.

Así, por ejemplo, la declaración de los derechos humanos del segundo congreso de demócratas de 1848 en Berlín afirmó los derechos siguientes:

a) El cuidado por sostener la existencia y libertad propias como derechos humanos fundamentales.

b) La inviolabilidad de la seguridad, libertad y propiedad de las personas, inclusive las relaciones comerciales.

c) El compromiso de la sociedad de cuidar la existencia de todos sus miembros a través de garantizar el trabajo o los medios de existencia, en caso de quedar inválido. 
d) Poner a la disposición los medios necesarios de apoyo para aquellos que carecen de recursos para sobrevivir.

Para el 24 de octubre de 1998- con ocasión de 150 aniversario de esta declaración se planificó el Tercer Congreso de demócratas en Berlín. Su tema central se tituló "Los perdedores de la Revolución de 1848-49. Los vencedores de hoy, exigencias de derechos humanos de 1848 , ayer y hoy".

Digitalizado por Biblioteca "P. Florentino Idoate, S.J." Universidad Centroamericana José Simeón Cañas 